\title{
NSF's support for education and training of the optics and photonics workforce
}

Carmiña Londoño, Richard Nash, Amelia Greer

Carmiña Londoño, Richard Nash, Amelia Greer, "NSF's support for education and training of the optics and photonics workforce," Proc. SPIE 11143, Fifteenth Conference on Education and Training in Optics and Photonics: ETOP 2019, 1114305 (2 July 2019); doi: 10.1117/12.2532497 Photonics: ETOP 2019, 2019, Quebec City, Quebec, Canada 


\title{
NSF's Support for Education and Training of the Optics and Photonics Workforce
}

\author{
Carmiña Londoño, Richard Nash, Amelia Greer \\ National Science Foundation, 2415 Eisenhower Avenue, Alexandria, VA USA 22314
}

\begin{abstract}
The National Science Foundation (NSF) is committed to creating an educated and skilled workforce in optics and photonics at all levels from K-12 students to Associate Degrees and PhDs through a variety of decentralized funding opportunities as described in this paper and associated presentation. NSF has special funding mechanisms for teachers through the Research Experiences for Teachers (RET) program and for undergraduate students through the Research Experiences for Undergraduates (REU) program. NSF reaches out to community colleges to support a variety of associate-level programs for technicians through its Advanced Technology Education (ATE) program. Lastly through multi-institutional and multidisciplinary ten-year investments via the Engineering Research Centers (ERC) program, NSF is preparing a cadre of next-generation optics and photonics engineers and scientists with the technical knowledge and entrepreneurial skills to address critical global challenges and to succeed in a competitive and interconnected world.
\end{abstract}

Keywords: Research Experiences for Teachers, Research Experiences for Undergraduates, Advanced Technology Education, Engineering Research Centers, National Science Foundation

\section{INTRODUCTION}

NSF has several funding mechanisms and programs to support the education and training of the optics and photonics U.S. workforce. These programs are not centralized and can be found throughout the Foundation. They vary in extent from single investigator research awards that usually cover the expenses of graduate students who advance particular research areas, to large multi-institutional Centers and infrastructure investments that help train the next generation's workforce.

\subsection{NSF Background}

The National Science Foundation (NSF) is an independent federal agency created by Congress in 1950 "to promote the progress of science; to advance the national health, prosperity, and welfare; to secure the national defense...." NSF has an annual budget of approximately \$8 billion dollars (FY 2019), and is the funding source for approximately 27 percent of the total federal budget for basic research conducted at U.S. colleges and universities. NSF is the major source of federal funding for basic research in many fields including mathematics, computer science, and the social sciences.

Currently NSF makes about 12,000 new awards per year, with an average duration of three years, to fund specific research proposals that have been judged the most promising by a rigorous and objective merit-review system. Most of these awards go to individuals or small groups of investigators. Others provide funding for research centers, instruments, and facilities that allow scientists, engineers, and students to work at the outermost frontiers of knowledge. Figure 1 summarizes NSF proposal and award outcomes information for FY 2018. 


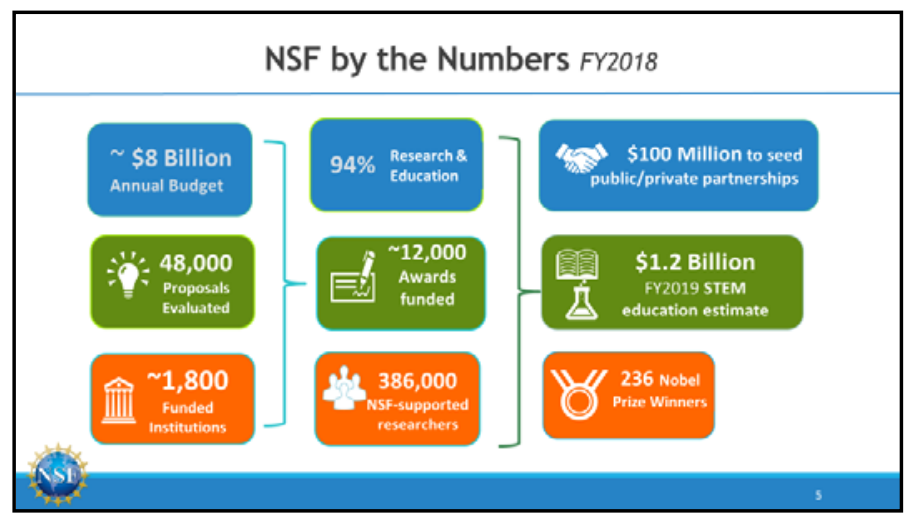

Figure 1. NSF by the numbers for FY 2018

In the past several decades, NSF-funded researchers have won 236 Nobel Prizes. ${ }^{1}$ These pioneers have included the scientists or teams that discovered many of the fundamental particles of matter, analyzed the cosmic microwaves left over from the earliest epoch of the universe, developed radiometric dating of ancient artifacts, decoded the genetics of viruses, and discovered an entirely new state of matter called a Bose-Einstein condensate.

NSF also funds equipment that is needed by scientists and engineers but is often too expensive for any one group or researcher to afford. Examples of such major research investments include giant optical and radio telescopes, Antarctic research sites, high-end computer facilities and ultra-highspeed connections, ships for oceanographic research, sensitive detectors of very subtle physical phenomena, and gravitational wave observatories.

Another essential element in NSF's mission is support for science and engineering education, from pre-K through graduate school and beyond. The research NSF funds is thoroughly integrated with education to help ensure that there will always be plenty of skilled people available to work in new and emerging scientific, engineering and technological fields, and plenty of capable teachers to educate the next generation. 


\section{DECENTRALIZED NSF FUNDING FOR OPTICS AND PHOTONICS}

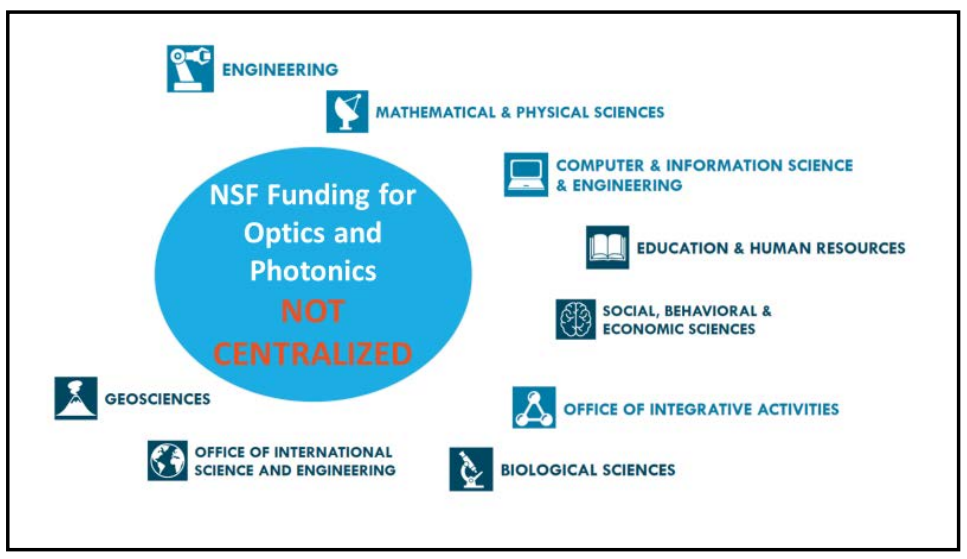

Figure 2. NSF funds O\&P throughout all its Directorates

Because the optics and photonics fields are inherently interdisciplinary in nature, often requiring expertise across traditional disciplinary boundaries, NSF funding for O\&P is supported mainly through core programs residing across all NSF Directorates, see Figure 2, with principal O\&P funding taking place in the Directorates for Engineering (ENG), Mathematical and Physical Sciences (MPS), and Education and Human Resources (EHR). A brief description of some of the most important programs in these three Directorates follows.

\subsection{NSF Engineering Directorate (ENG)}

In ENG there are several programs that directly support the optics and photonics communities. For example, the Biophotonics program funds fundamental engineering research for new technologies in medical diagnostics and therapies. The Electronic, Photonic, and Magnetic Devices (EPMD) program supports a wide range of optics and photonics research and education activities. Areas of interest in EPMD include: improving fundamental understanding of optoelectronic devices based on the principles of nonlinear optics, quantum optics, or ultrafast photonics. Also of interest are novel photonic devices based on organic, inorganic, or hybrid materials on flexible substrates, metamaterials and plasmonics, and emerging 2D atomic-layered materials as well as topological photonic structures. The program also aims to advance the frontiers from Extreme Ultraviolet (EUV) to Terahertz (THz) in optical sources (such as high power, tunable, broadband, frequency comb and ultrafast lasers and arrays) and photodetectors. Finally, EPMD also supports research in novel optical communication devices, photonic integrated circuits, single-photon quantum devices, and nano-photonics.

The Engineering Research Centers (ERCs) program funds interdisciplinary centers located at universities across the entire United States that provide an environment where academia and industry can collaborate in pursuing strategic advances in complex engineered systems and systems-level technologies. Activity within ERCs lies at the interface between the discovery-driven culture of science and the innovation-driven culture of engineering. There have been several ERCs focused 
primarily on optics and photonics, which have educated many hundreds of students in the field, please see section 5.1 .

\subsection{NSF Mathematical and Physical Sciences Directorate (MPS)}

The optics and photonics program in MPS explicitly addresses research proposals that are relevant either to one discipline represented in MPS or lying at the interface of applied mathematics, statistics, and the core science disciplines of astronomy, chemistry, physics, mathematics, and materials research. Priority research areas at MPS for optics and photonics include:

- Plasmonics and nanophotonics: controlling optical fields and propagation on the nanoscale

- Coherent electromagnetic fields: attosecond time scales and x-ray photon energies

- Optomechanical interactions: from single-molecule mechanics to macroscopic quantum state

- $\quad$ Seeing beyond the diffraction limit and new imaging modalities

- Creating and controlling quantum coherence with light

- Controlling molecules with light and light with molecules

- Observing the universe: optics and photonics for astronomy and astrophysics

Examples of specific call for proposals that impact optics and photonics in MPS include:

1. The Advanced Technologies and Instrumentation program which funds the development and construction of state-of-the-art astronomical detectors and instruments for the visible, infrared, submillimeter, and radio regions of the spectrum.

2. The Chemical Measurement and Imaging program funds research on chemically-relevant measurement science and imaging including the development of new chemical imaging and measurement tools that probe chemical properties and processes.

3. The Electronic and Photonic Materials program focuses on novel materials and their integration that may offer new paradigms in critical computing, communication and sensing components, or enable advanced optoelectronics and photonics

4. The Atomic, Molecular, and Optical Experimental Physics program focuses on the fundamental quantitative understanding of atoms and molecules and their interaction with light.

5. The Materials Research Science and Engineering Centers support materials research and education by fostering active collaboration between academia and other sectors that enable researchers to address problems of a scope and complexity requiring the advantages of scale and interdisciplinarity provided by a campus-based research center.

6. The Physics Frontiers Centers program supports university-based centers with the potential for profound advances in physics and also include creative, substantive activities aimed at enhancing education, diversity, and public outreach.

\subsection{NSF Education and Human Resources Directorate (EHR)}

In EHR one of the most relevant programs to the optics and photonics workforce is the Advanced Technological Education (ATE) program, which focuses on two-year Institutions of Higher Education (IHEs), and on the education of technicians for the technically advanced fields that drive our nation's economy. The program involves partnerships between academic institutions (grades 712, IHEs) and industry to promote improvement in the education of science and engineering technicians at the undergraduate and secondary-institution school levels. The ATE program supports 
curriculum development, professional development of college faculty and secondary school teachers, career pathways, and other activities. The program invites research proposals that advance knowledge related to technical education. NSF anticipates that approximately $\$ 66$ million will be available for new and continuing awards in this program in FY 2019.

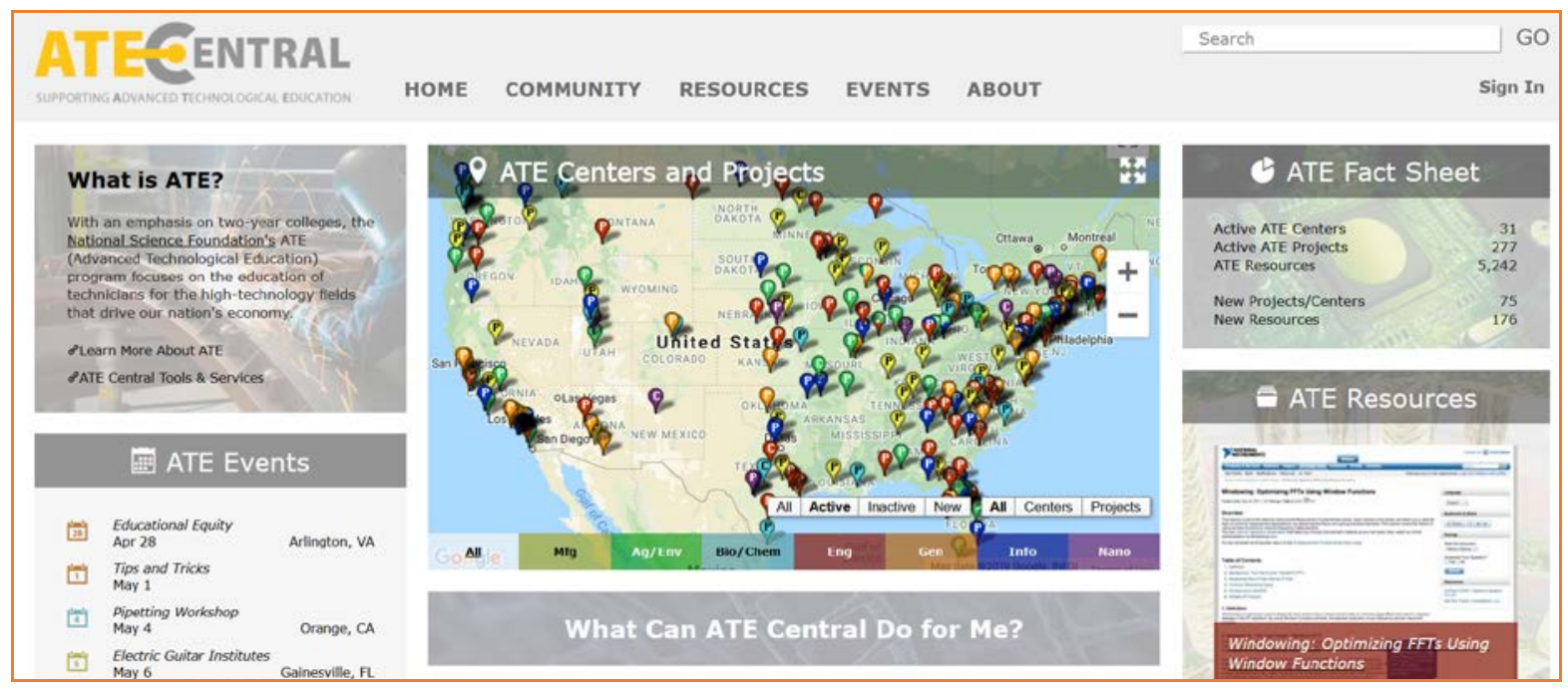

Figure 3. ATE Central webpage.

The ATE program supports proposals in a variety of tracks including Projects, Centers, and Targeted Research in Technician Education. For more information about the ATE program, please visit the resources listed below:

1. The ATE home page on the NSF website provides an overview of the ATE program, contact information for the associated program officers, and examples of recent awards. ${ }^{2}$

2. The ATE solicitation provides detailed information about the program and outlines key concerns of the funding agency, budgetary guidelines, and programmatic requirements. ${ }^{3}$

3. The ATE@20 Book outlines the first 20 years of the ATE program, providing history, stories, and pictures that highlight the impact of ATE grantees nationwide. ${ }^{4}$

4. The ATE@20 Blog provides further coverage of stories that showcase ATE accomplishments, highlights news and events of interest to the community, and provides a dissemination pathway for ATE impacts. ${ }^{5}$

5. Visit ATE Central's Stay In the Loop page to keep abreast of community activities and news. ${ }^{6}$

\section{RESEARCH EXPERIENCE FOR UNDERGRADUATES (REU)}

NSF funds many research opportunities for undergraduate students through its REU Sites program. An REU Site consists of a group of approximately ten undergraduates who work in the research programs of a host institution. Each student is associated with a specific research project in which he/she works closely with faculty and other researchers. Students are granted stipends and in many cases receive assistance with housing and travel. Undergraduate students supported with NSF funds must be citizens or permanent residents of the United States or its territories. An REU Site may be at either a U.S.-based or foreign location. 
Those interested are urged to search through the REU Site webpage to examine opportunities in the subject areas supported by various NSF Directorates. Also, keyword searches can be used to identify sites in particular research areas or locations. Students must contact the individual sites for information and application materials. NSF does not provide application materials and does not select student participants. A contact person and contact information is listed for each site.

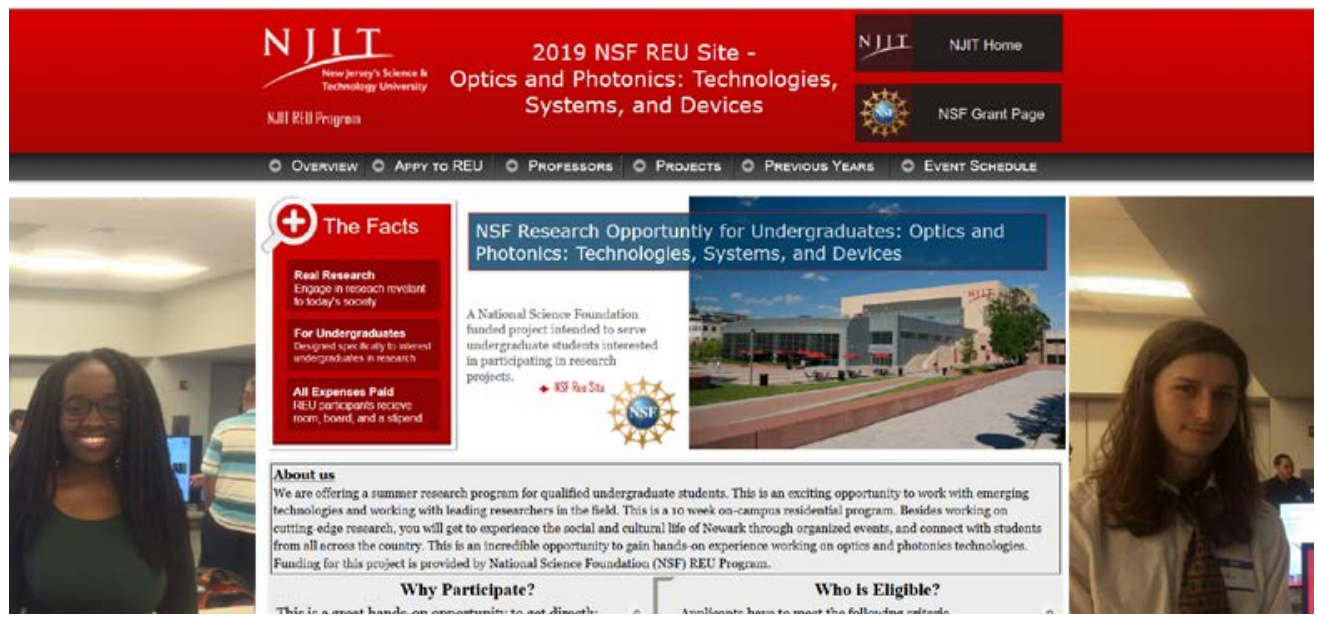

Figure 4. New Jersey Science \& Technology (NJIT) REU Program

As shown in Figure 4 one example of an REU Site is the 2019 New Jersey Science \& Technology (NJIT) REU Program titled "Optics \& Photonics: Technologies, Systems, and Devices." This is a great hands-on opportunity to get directly immersed in active research by working closely with the research faculty at NJIT. Through this program, students gain experience in the design, implementation, and evaluation of a research project, as well as develop knowledge of opportunities within the optics and photonics field. In addition, each participant receives a $\$ 4,500$ stipend, oncampus housing, meals, and compensation for travel expenses to and from the REU Site. There are ten topics to choose from including projects on: Infrared Photoconductive Photodetectors Based-on Colloidal Semiconductor Nanocrystal; Optical characterization of rat brain tissue after injury; IIINitride Nanowire Deep Ultraviolet Light-Emitting Diodes for Precise Applications; Optimization and Characterization of Neuromorphic Devices; Indoor Visible-light Communication test-bed and simulator. Over the past ten years, at least 38 REU Aites have featured research in optics and photonics.

\section{RESEARCH EXPERIENCE FOR TEACHERS (RET)}

NSF's Directorate for Engineering (ENG) and the Directorate for Computer and Information Science and Engineering (CISE) have joined to support the NSF Research Experiences for Teachers (RET) in Engineering and Computer Science program which brings science, technology, engineering, computer and information science, and mathematics (STEM) K-12 teachers and community college faculty into the university research community. ${ }^{7}$

For at least six weeks during the summer, RET participants conduct engineering and/or computer science research in university labs and facilities, which leads to the transfer of new knowledge to 
classroom activities. In addition, during the academic year there is ongoing interaction between participating university faculty and RET participants to ensure that the research experience is translated into classroom practice and that the lesson plans, curricula, and other skills developed during the summer are implemented in the classroom.

The research projects and experiences all revolve around a focused area related to engineering and/or computer science. The K-12 STEM teachers and/or full-time community college faculty also translate their research experiences and new scientific knowledge into their classroom activities and curricula. The university team includes faculty, graduate and undergraduate students, as well as industrial advisors. Involvement of graduate students in support of academic-year classroom activities is particularly encouraged. Partnerships with inner-city, rural or other high-needs schools are especially encouraged, as is participation by underrepresented minorities, women, veterans, and persons with disabilities.

The RET program features two mechanisms for support of in-service and pre-service K-12 STEM teachers and full-time community college faculty: (1) RET supplements to ongoing ENG and CISE awards and (2) new RET Site awards. RET supplements may be included for new or renewed ENG and CISE grants or as supplements to ongoing ENG- and CISE-funded projects. In the past ten years, 11 RET Sites have featured research in optics and photonics.

\section{ENGINEERING RESEARCH CENTERS PROGRAM (ERC)}

\subsection{ERC Background}

NSF created the Engineering Research Centers (ERC) program in 1984 to bring technology-based industry and universities together to strengthen the competitive position of the United States in the global marketplace. These partnerships established cross-disciplinary Centers focused on advancing fundamental engineering knowledge and engineered systems technology while exposing students to the integrative aspects of engineered systems and industrial practice. The present-day ERC program supports convergent research that leads to strong societal impact. Each ERC must have four interacting foundational components, see Figure 5, that are connected by an integrated, holistic ERC vision and strategic plan. ERCs are expected to go beyond the research project, including engineering workforce development at all participant stages, a culture of diversity and inclusion where all participants gain mutual benefit, and value creation within an innovation ecosystem that will outlast the ten-year lifetime of the ERC. 


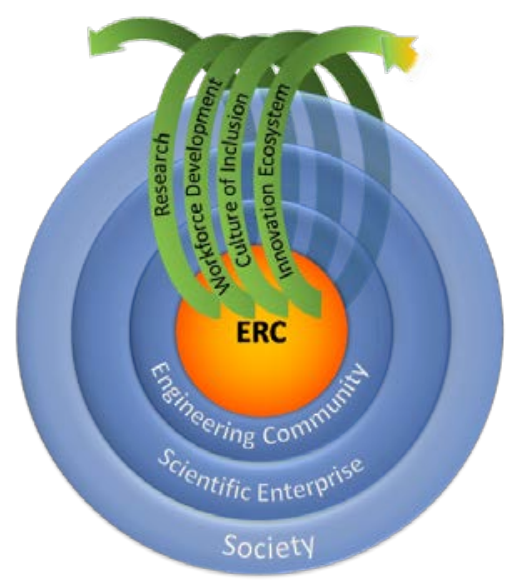

Figure 5. Engineering Research Centers make a dynamic impact on society in several ways.

\subsection{Engineering Workforce Development (EWD)}

Workforce Development is expected to occur at all levels of the ERC and providing opportunities for all ERC members including students, faculty, and external partners as appropriate. The ERC must have an evidence-based program for building human capacity for the future engineering workforce. The PIs leading an ERC need to provide strategic goals for the ERC as well as targeted and specific outcomes related to workforce development and education. The activities should logically lead to targeted outcomes and support diverse pathways and experiences for participants. At least 6 non-ERC students must enroll in a REU program budgeted at a minimum of $\$ 42 \mathrm{~K}$ per year from the ERC base budget. Awarded ERCs are encouraged to submit proposals to the annual REU Site and RET Site competitions to expand the Center's workforce development impact. Partnerships with inner city, rural, or other high needs schools are especially encouraged, as is participation by underrepresented minorities, women, veterans, and persons with disabilities. Suitable metrics to assess progress towards meeting the ERC's goals must be included and feedback loops should be in place for program improvement. 


\subsection{ERCs focused on Optics and Photonics}

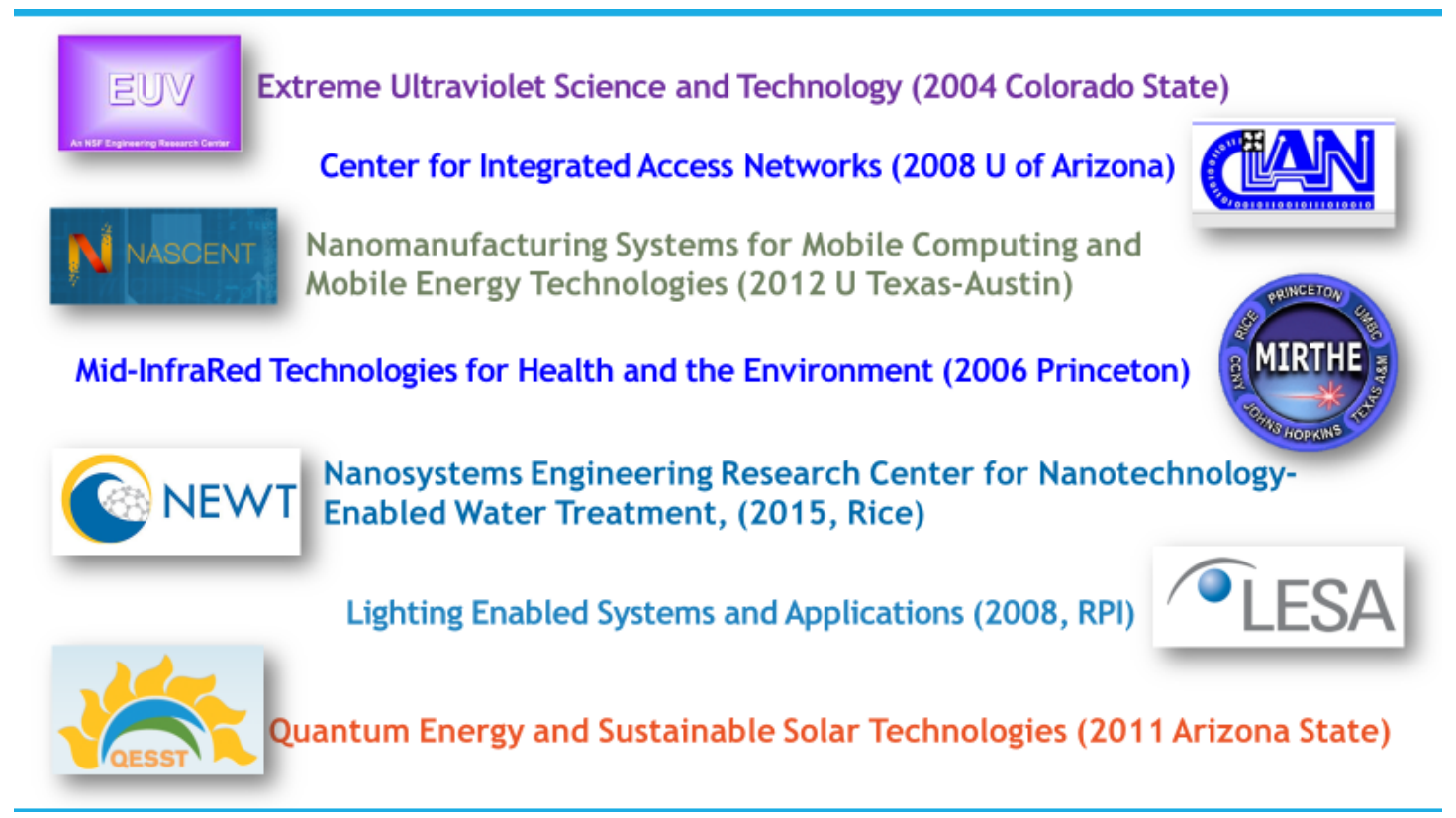

Figure 6. Engineering Research Centers focused on optics and photonics.

Through its 30 year history the NSF ERC Program has funded several ERCs that have focused primarily on optics and photonics. Figure 6 only lists the O\&P ERCs since 2004. These include the Center for Extreme Ultraviolet Science and Technology at Colorado State, Integrated Access Networks at the University of Arizona, Nanomanufacturing Systems for Mobile Computing and Mobile Energy Technologies at the University of Texas-Austin, Mid-InfraRed Technologies for Health and the Environment at Princeton University, Nanosystems Engineering Research Center for Nanotechnology-Enabled Water Treatment at Rice University, Lighting Enabled Systems and Applications at Rensselaer Polytechnic Institute, and the Quantum Energy and Sustainable Solar Technologies at Arizona State University. The number of students that trained in optics and photonics (and who are presently being trained) by these ERCs exceeds many hundreds. The reader is urged to visit the websites of these ERCs to read about the programs and opportunities offered to students.

\section{CONCLUSIONS}

NSF is committed to creating an educated and skilled workforce in optics and photonics. We have discussed several mechanisms which NSF uses to support the O\&P community emphasizing its decentralized nature. As shown in Figure 2, all the NSF Directorates fund in one way or another optics and photonics. The reader is urged to consult the NSF webpage to search for other NSF programs that support optics and photonics but were not described here. 


\section{REFERENCES}

[1] “The Nobel Prizes: The NSF Collection.” NSF 2019.

https://www.nsf.gov/news/special_reports/nobelprizes/index.jsp

[2] “Advanced Technological Education (ATE).” NSF 2019.

https://www.nsf.gov/funding/pgm_summ.jsp?pims_id=5464

[3] “Advanced Technological Education (ATE): Program Solicitation NSF 18-571.” NSF 2019.

https://www.nsf.gov/pubs/2018/nsf18571/nsf18571.htm

[4] “About the ATE@20 Book.” ATE 2019. https://atecentral.net/ate20/book

[5] "ATE Impacts.” ATE 2019. https://atecentral.net/impacts/

[6] “ATE Central.” ATE 2019. https://atecentral.net/intheloop

[7] "Research Experiences for Teachers (RET) in Engineering and Computer Science.” NSF 2019.

https://www.nsf.gov/funding/pgm_summ.jsp?pims_id=505170

Proc. of SPIE Vol. $111431114305-10$

Downloaded From: https://www.spiedigitallibrary.org/conference-proceedings-of-spie on 26 Apr 2023 Terms of Use: https://www.spiedigitallibrary.org/terms-of-use 